METROLOGY AND MEASUREMENT SYSTEMS
Index 330930, ISSN 0860-8229
www.metrology.pg.gda.pl

\title{
BIOMETROLOGICAL METHOD OF PELVIS MEASUREMENT AND ANATOMICAL POSITIONING OF ENDOPROSTHESIS OF HIP JOINT
}

\author{
Andrzej Ryniewicz ${ }^{1)}$, Anna Maria Ryniewicz ${ }^{2,3)}$, Tomasz Madej $^{2)}$, Jerzy Sładek ${ }^{1)}$, \\ Adam Gąska ${ }^{1)}$ \\ 1) Cracow University of Technology, Faculty of Mechanical Engineering, Aleja Jana Pawla II 37, 31-864 Kraków, Poland \\ (andrzej@ryniewicz.pl, sladek@mech.pk.edu.pl, \agaska@mech.pk.edu.pl, +48 12628 3230) \\ 2) AGH University of Science and Technology, Faculty of Mechanical Engineering and Robotics, Mickiewicza 30, 30-059 Kraków, \\ Poland(madej@imir.agh.edu.pl) \\ 3) Jagiellonian University Medical College, Faculty of Medicine, Montelupich 4, 31-155 Kraków, Poland (anna@ryniewicz.pl)
}

\begin{abstract}
This elaboration presents the method of virtual positioning of the construction of an endoprosthesis of hip joint in a patient's pelvis and femoral bone, reconstructed on the basis of imaging obtained from computer tomography. It is based on the matching of an implant to individual anatomical-biomechanical conditions. The method is established on the following procedures: diagnostic, spatial modeling, virtual measuring and targeted biometrological application for the model of bone structures. The final effect of the completed procedures is selection and optimal positioning of the endoprosthesis of hip joint before a planned medical intervention. The determined geometrical parameters of bone structures and settled positioning of the endoprosthesis can create data for the system of computer navigation.
\end{abstract}

Keywords: measurement, solid modeling, endoprosthesis of hip joint, pelvis, virtual positioning.

(C) 2013 Polish Academy of Sciences. All rights reserved

\section{Introduction}

The alloplasty operation of a hip joint by the introduction of a specified interchangeable head on the stem and the cup changes completely the conditions of the cooperation characteristic for the biological proper pair. Because of the different reaction of cooperating elements of the endoprostheses and different reaction on the surrounding tissues, the handicap of the biolubrication and the disturbance of the relation of stresses and displacements in the biobearing occur. The reasons for the loss of the functionality of the artificial joint are very complex and possible to be estimated only in the further prognosis $[1,2,6]$. That is why the authors were forced to develop a method which can indicate the optimal location of the endoprosthesis adapted to the patient's anatomical conditions before the operation.

\section{Materials and methods}

The material used to elaborate and verify the method is comprised of targeted imaging tests carried out by the computer tomography and accomplished in a horizontal position for the total alloplasty of a hip joint. The location of the endoprosthesis in bone structures decides on the conditions of exploitation of an artificial joint [6, 10-13]. Considering the occurrence of individual differences in the osteoarticular system of the pelvis and femoral bone, their identification becomes the basic issue before the implantation operation of the hip joint $[7,11]$. 
The aim of the presented method is the virtual reconstruction of the pelvic girdle, the estimation of bone tissue, linear and angular measurements in the established reference system and formulation of directives for selecting the endoprosthesis in the shape-material and functional range. The next step is the selection of construction of endoprostheses from the base of implants admitted to the orthopedic application. This selection takes into consideration accordingly: the diameter and depth of the anatomical acetabulum, the angle of inclination and turn of this acetabulum, neck-stem angle of the femoral bone as well as the light of the marrow canal in the femoral bone. The chosen constructions or one construction of the endoprosthesis are modeled in 3D. The final effect of these executed procedures is the ultimate selection and targeted biometrological application of positioning the implant in the patient's pelvis and femoral bone according to the determined anatomical-geometrical parameters and biomechanical conditions.

The data comprising the global numerical model with the implanted endoprosthesis can be introduced to the system of computer navigation $[3,4]$. It can also be used to the strength verification where the distributions of stresses and displacements in the loaded model can be determined by different variants of locomotion $[10,12,13]$.

The next stages of this developed method are presented in the procedure diagram of positioning the endoprosthesis of the hip joint in the patient's anatomical structures (Fig. 1).

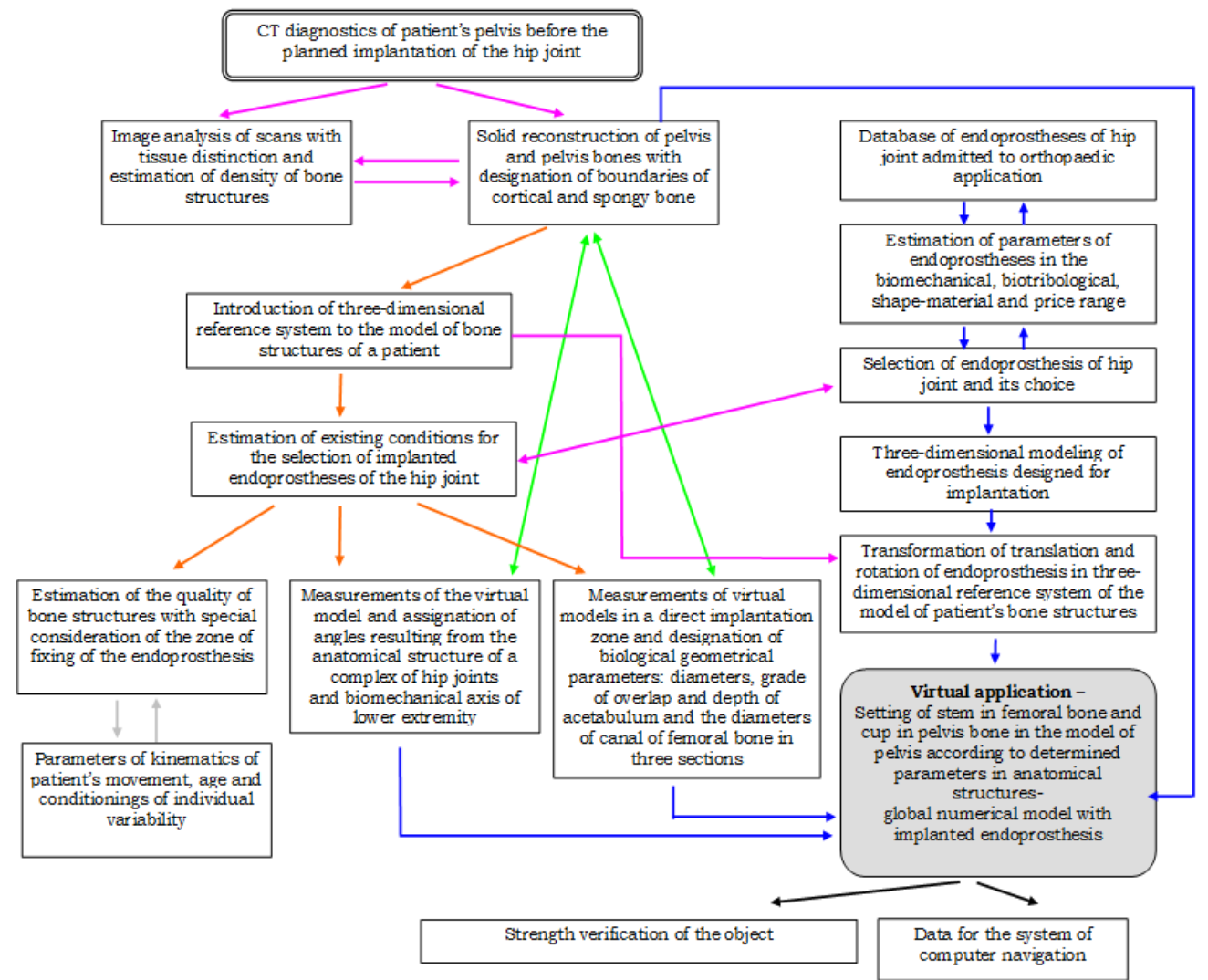

Fig. 1. The procedure diagram of positioning the endoprosthesis of the hip joint in the patient's anatomical structures. 


\subsection{Modeling of bone structures}

Due to the great number of the constructional-material solutions of endoprostheses and the fundamental dilemma concerning the selection of the tribological pair for an individual patient, a method of preclinical tests was elaborated using solid modeling [10, 12, 13].

This method enables the complex analysis of a numerical object created taking into account the tomographical representation of the patient's bone structures (Fig. 2-5), threedimensional modeling of the construction of the endoprosthesis (Fig. 6 and 7) and virtual application and positioning of a specific solution in the anatomical system (Fig. 8, 10-13).

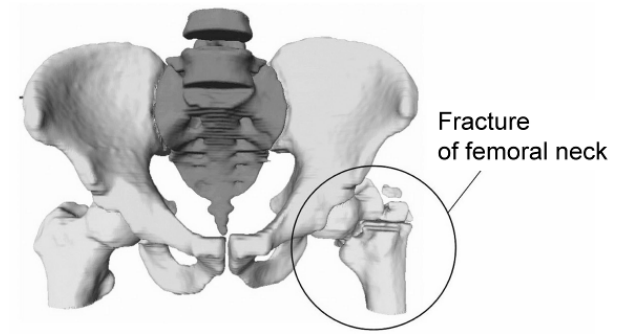

Fig. 2. Three-dimensional representation of a patient's pelvic girdle with a fracture in the area of the left neck of the hip joint, on the basis of CT diagnostics and image analysis in Amira software.

a)

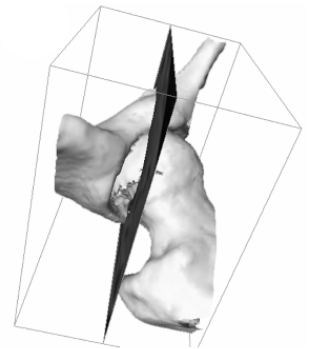

b)

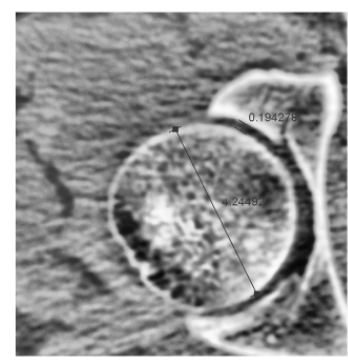

Fig. 3. Reconstruction of the hip joint: a) marked plane of a section; b) measured axial section.

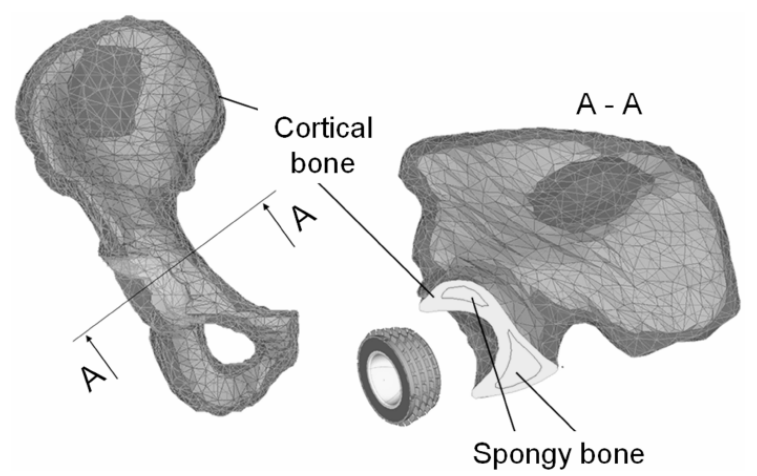

Fig. 4. Virtual application of a cup to the structures of the pelvic bone. 
a)

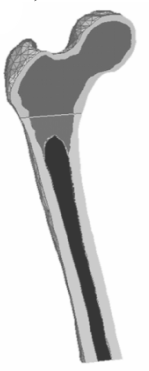

b)

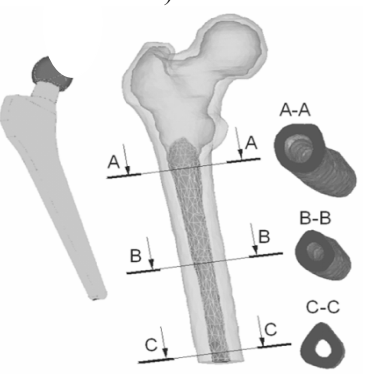

Fig. 5. Virtual sections through the bone marrow canal of the patient's femoral bone: a) longitudinal; b) transverse.

From the centers that cooperate in the project, CT measurements of diagnosed and treated patients taken in accordance to the special procedure were obtained. The obtained sequences of scans from the area of imaging of the pelvis were subjected to graphic processing using Amira, the software for image analysis, spatial reconstruction and object visualization. The analysis of the levels of greyness with the differentiation of the degree of absorption of X-ray according to the Hounsfield scale was conducted for the particular images in the planes of scanning. In the reconstruction, bone structures of the pelvis, in which boundaries between the cortical bone, spongy bone and the marrow canal were determined (Fig. 4), and of femoral bones, in which the cortical bone, spongy bone and marrow canal were distinguished (Fig. 5). The qualitative and geometrical parameters of the patient's distinguished tissues had basic meaning for the application and positioning of the cup and stem of the endoprosthesis. In the accomplished reconstructional projections and biomeasurements, the quality and measurement uncertainty were determined $[12,13]$.

In the elaborated method, it is preferable to take advantage of the relation between the density of the bone tissue and its strength parameters, because the correlation between the darkening of the CT images and the density of the bone tissue was confirmed. The density of the patient's bone tissue is of essential meaning for the stabilization of the implant in these structures, both for cemented and cementless endoprostheses. In the case of a diagnosed patient, in consultation with the radiologist and orthopedist the density of bone structures were assessed in the context of osteoporosis. Osteoporotic changes were not found.

\subsection{Modeling of endoprosthesis of the hip joint}

The endoprostheses which have permission for clinical applications are produced in many material and geometrical configurations which makes it possible to match the patient's individual features. Nowadays, 36 types of cemented and cementless constructions of artificial joints are accessible. However, their selection is connected with a whole range of various conditions.

An orthopaedic surgeon realizes that the vitality of the endoprosthesis is determined by the quality and density of the patient's bone tissue.

The surgeon also faces the dilemma of whether to choose a cemented or cementless one and has to answer other questions: Which friction-wear connection will be the most suitable? Which endoprosthesis is better? The one in which the cooperating movement elements are hard, less flexible and of reduced emission of wear products or a different one having an increased amount of the pulp emitted to the surrounding tissues but of higher parameters that absorb and cooperate better with the tissues around the endoprosthesis. 
After that, further aspects must be taken into consideration. The characteristics of the patient's locomotion must be diagnosed together with the determination of the biomechanical axis of the lower limb.

Finally, geometrical sizes of the elements of the endoprosthesis must be matched, which at the same time must be adjusted to the anatomical shapes in the zones of its fixation.

To realize the project, a virtual bank of endoprostheses of the hip joint of various constructional-material solutions was founded. The solid modeling based on Boolean algebra and the program NE/Nastran Modeler were used to create the geometry of the layer cups, heads and stems of cemented and cementless endoprostheses [10,12,13].

All the modeled elements of the endoprosthesis enable the virtual generation of the bank of various constructional solutions of endoprostheses which are characterized by:

- in the area of cups - diversified constructional structure, different shapes and sizes (providing the locomotion functions and specified scopes of movement) - involving series of types which enable proper transfer of pressure from the construction to the tissues of the anatomical bed using biomaterials which are diversified in strength and tribological aspects.

- in the area of heads - diversification of sizes and shape toleration adjusted for the cooperation with the cup, diversified by the character and geometry of the connection with the stem and the type of the constructional biomaterial which protects the strength and tribological parameters.

- in the area of stems - of diversified shapes, length, straight and curved, right and left having different neck-stem angle, created from various biomaterials providing optimal contact with the femoral bone and the proper transfer of pressures on the carrying structures of the femoral bone.

From that bank of solutions for the particular types of endoprostheses, one can form the configuration in relation to the size and shape of natural and individually variable patient's bone structures as well as the required proper biomaterial associations (Fig. 6 and 7). In the elaborated system there is also a possibility for the configuration of the elements of endoprostheses from different producers, if necessary.

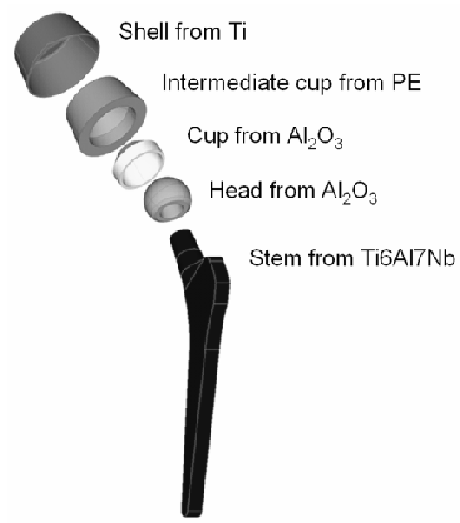

Fig. 6. Modeled exemplary constructional solution of cementless endoprostheses: stem from Ti6Al7 Nb, head from $\mathrm{Al}_{2} \mathrm{O}_{3} \varnothing 28 \mathrm{~mm}$, three-layered cup $\mathrm{Al}_{2} \mathrm{O}_{3}-\mathrm{PE}-\mathrm{Ti}$. 


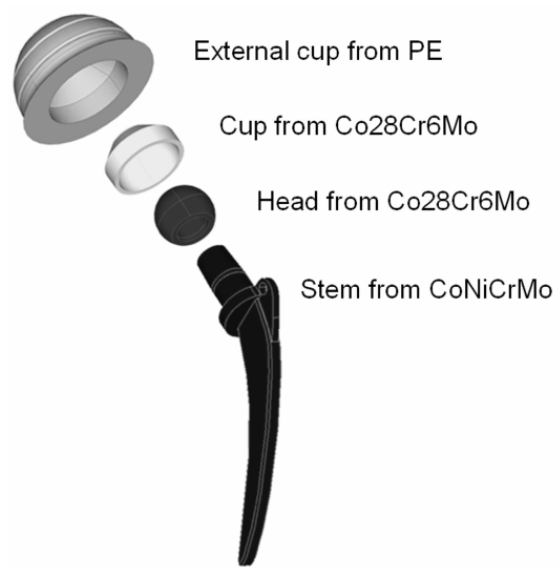

Fig. 7. Modeled exemplary constructional solution of cemented endoprostheses: stem from CoNiCrMo, head from Co28Cr6Mo Ø28 mm, two-layered cup Co28Cr6Mo-PE.

\subsection{Positioning of the implant}

The tomographical representation of the pelvic girdle performed in Amira software was exported to the program NE/Nastran Modeler. In this environment, the virtual application of the endoprosthesis was conducted and that can be used in the alloplasty operations carried out by computer navigation $[3,4]$.

The positioning of the endoprosthesis were preceded by an analysis of the solid geometry of the patient's bone structures in the base plane system: transverse, horizontal and sagittal ones. Considering the accepted spatial system, using descriptive geometry, the characteristic linear dimensions of the biological acetabulum and femoral bone, angles of cup orientation in the pelvic bone and stem and head in the femoral bone. Solid modeling in NE/Nastran Modeler gives the opportunity to separate structures, their projections and rotations in the accepted reference system. Those operations, considering the solutions known from publications $[3,5,7,8,14]$, constitute the original solution of the problem of assessment of the cup and stem positioning in bone structures. These possibilities were used during measurements and positioning of the endoprosthesis of the hip joint in a reconstructed pelvic girdle.

Measurements of the diameter of the acetabulum were performed in a procedure of its projection onto the inclination plane created by the acetabulara limbus. The local coordinate system has been appointed in the way that the YZ plane is a plane created by the bone limbus while the $\mathrm{XZ}$ plane is built in the intersection plane of the acetabulum that passes its center. Biological acetabula were oval and their long axes were respectively $61,9 \mathrm{~mm}$ and $60,4 \mathrm{~mm}$ long (Fig. 8). The depth of the acetabulum and its inclination angle were determined by rotating the pelvic bone to a position which is perpendicular to the plane of its inclination which passes the center of the biological acetabulum (Fig. 8). The depth of the acetabulum amounted to $23 \mathrm{~mm}$.

The essential problem in the alloplasty of the hip joint is the proper spatial orientation of the cup component. According to various authors, the range of the angle of the cup's setting includes in large limits from $30^{\circ}$ at McKee to $60^{\circ}$ at Engh $[1,2]$. 

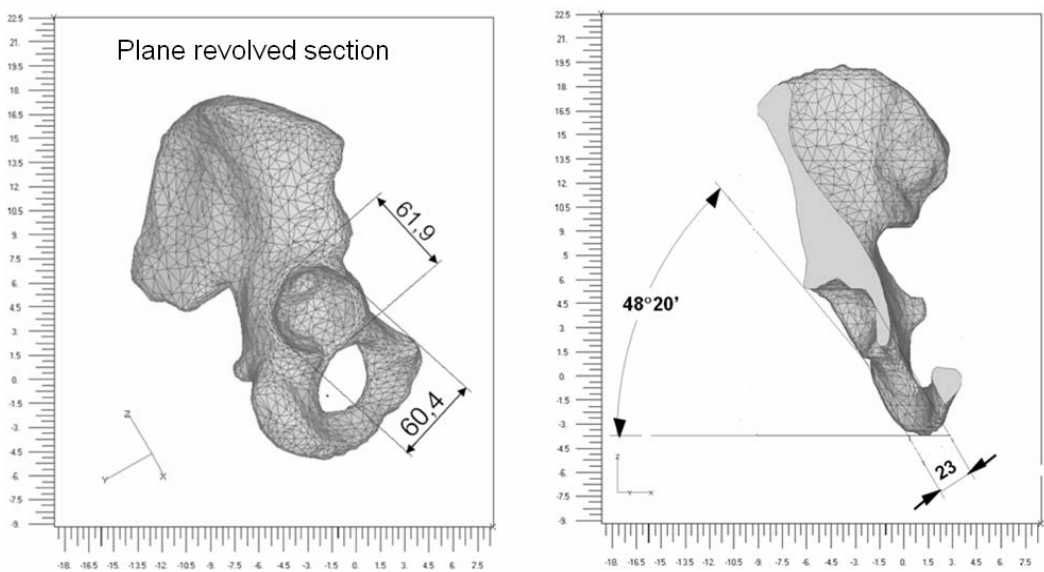

Fig. 8. Determination of dimensions and inclination angle of a biological acetabulum.

Charnley introduced the standard $45^{\circ} \pm 10^{\circ}$, which is supported by many authors, as proper cups, $56^{\circ}$ and more as vertical cups and $34^{\circ}$ or less as horizontal ones (Fig. 9). Its turn is equally as important as the inclination of the cup, which should amount from 0 to $5^{\circ}$ according to Charnely, and from 0 to $10^{\circ}$ according to Judet, Kreczko and Wejslog. The location of the cup is thought to be optimal if the biomechanical center of the artificial joint is not dislocated over $5 \mathrm{~mm}$ towards the center of the second proper joint.

The three-dimensional reconstruction and solid modeling of the pelvis allowed to determine both angles of three-dimensional location of the cup [1, 6, 7, 9]. After their determination the chosen and modeled cup were applied. The cup was introduced in such a way that the bone bed of the biological acetabulum was saved. In the application procedure the plane formed by the acetabulara limbus and the horizontal and transverse planes were used. The inclination angle of the cup were determined as the angle between the vector perpendicular to the plane of the bone limbus and the vector perpendicular to the horizontal plane (Fig. 8). This angle amounted to $48^{\circ} 20^{\prime}$.

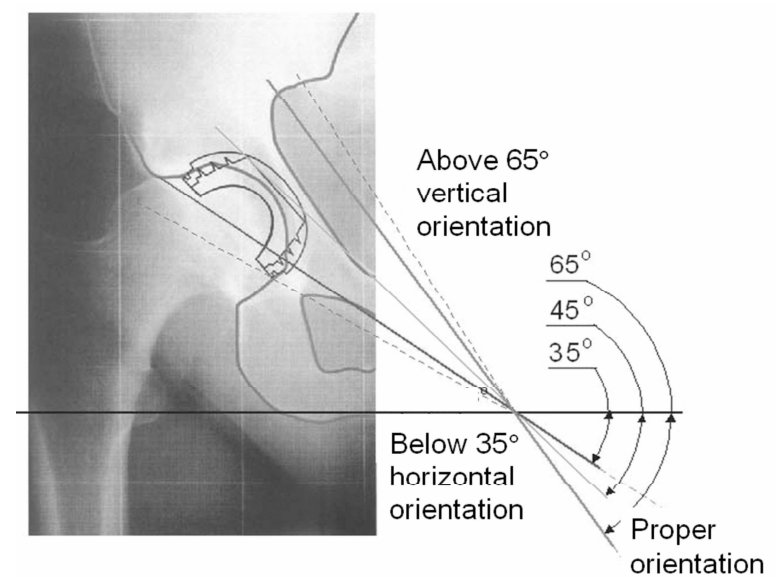

Fig. 9. Proper positioning of the cup in the girdle in the pelvis according to Charney [2]. 
The determination of the anteversion angle of the cup also came from the anatomical location of the acetabulum. This angle was determined between the axis of the acetabulum that is perpendicular towards the plane of the bone limbus and the transverse plane. The anteversion angle was measured in the plane determined by two lines: the cup axis and its projection on the transverse plane (Fig. 10). In the analyzed case that angle equalled $14^{\circ} 40^{\prime}$.

In the described case, the determined values of angles of inclination and anteversion of the cup were located inside the safe zone which equals $40^{\circ} \pm 10^{\circ}$ for the inclination angle and $15^{\circ} \pm 10^{\circ}$ for the anteversion angle and are suggested in $[1,8]$. The proposed procedure of angle determination is optimal regarding its accuracy and possibility of anatomical positioning of the cup. The migration of cups that were diagnosed in the post-intervention examinations may be due to difficulties and inaccuracies of cup mounting in the pelvic bone during the operation.

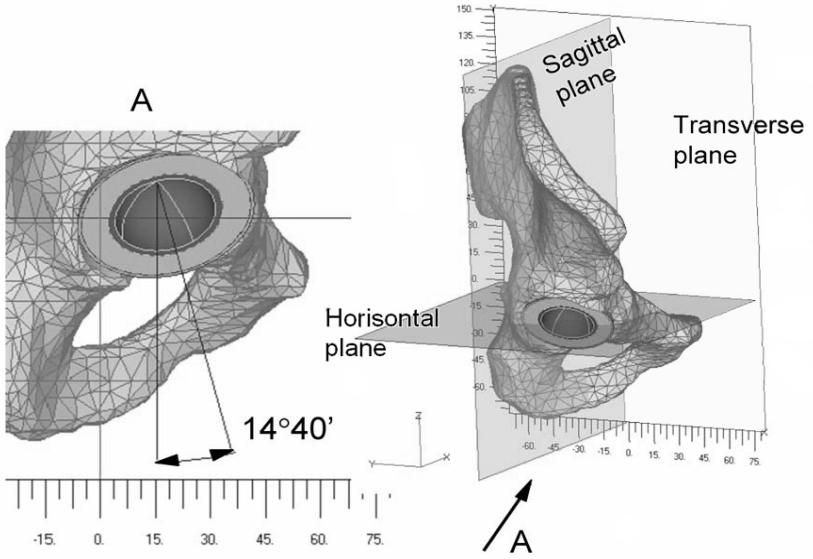

Fig. 10. Determination of the anteversion angle of the biological acetabulum as well as the setting of the cup of the implant keeping the inclination and anteversion angles.

The next important problem in the alloplasty of the hip joint is the selection and positioning of the stem of the endoprosthesis $[12,14,15]$. The geometrical parameters of the stem were determined basing on the shape identification of the femoral bone canal and biological neck-stem angle (Fig. 11). The parameters of the canal were measured in three parallel planes placed below trochanter minor. In marked intersections the mean values of the canal diameter were respectively: $\varnothing_{\mathrm{A}}=12,4 \mathrm{~mm} \varnothing_{\mathrm{B}}=8,2 \mathrm{~mm}, \varnothing_{\mathrm{C}}=6,8 \mathrm{~mm}$. The neck-stem angle was determined between the axis of the neck and the axis of the stem of the femoral bone (Fig. 11). In the case of the diagnosed patient this angle equaled $127^{\circ}$.

The second issue in the proper functioning of the endoprosthesis is keeping the antetorsion angle of the neck of the femoral bone forward, which is correlated with the inclination and anteversion of cup. The antetorsion angle of the neck is described in such a way that the proximal and distal extremities of the femoral bone are projected one to the other on the horizontal plane. This angle is determined by the axes of the neck and of the knee joint in the projection on the horizontal plane (Fig. 12). In the case of the diagnosed patient this angle equaled $12^{\circ} 30^{\prime}$ and was formed at the positioning of the stem and head of the endoprosthesis of the femoral bone.

After the creation of the virtual implants and their positioning in the pelvis and in the proximal parts of the femoral bones, complex solid models (Fig. 13) were obtained with the possibility to have a control conduct of the geometrical analyses. 

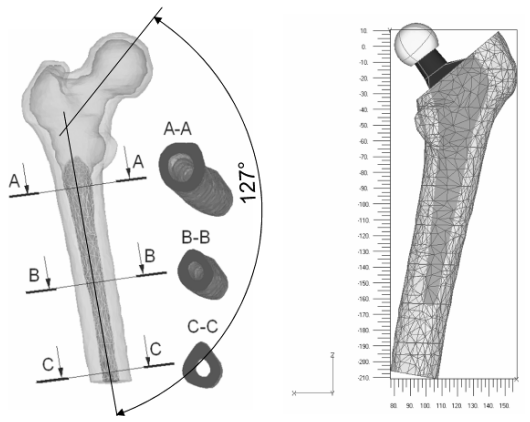

Fig. 11. Determination of the neck-stem angle and selection of the stem and the head of the implant.
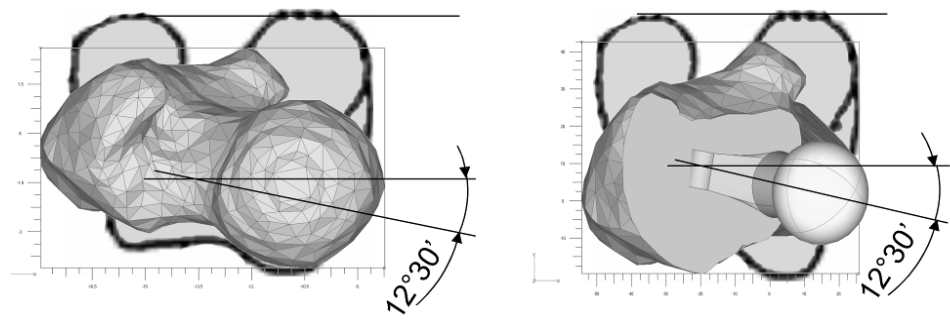

Fig. 12. Determination of the antetorsion angle of the neck of the femoral bone and establishment of the stem of the implant in the position corresponding to the neck-stem and the antetorsion angles.

a)

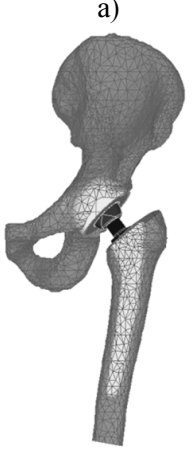

b)

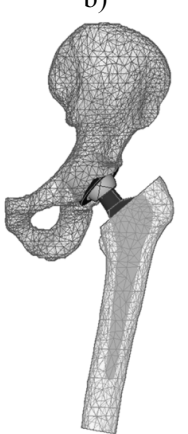

Fig. 13. Virtual setting of the endoprosthesis of the hip joint in bone structures of an individual patient in received reference system: a) cemented; b) cementless.

\section{Summary}

The operation of alloplasty of the hip joint is executed from the lower surgical approach and the operator cannot indicate other alternative possibilities in the selection and adjustment of endoprostheses to individual anatomical parameters. Standard X-ray diagnostics does not give such possibility either.

Supporting the implantation operation using CT, solid modeling, biometrology and positioning of endoprosthesis in the virtual bone structures allow to optimize the selection and such location which will guarantee the biomechanical axis of the limb, biomechanical parameters in the range of the bone bed and depth of the cup, angles of inclination and 
anteversion of the cup, light of the canal of the femoral bone, angle of neck-stem and antetorsion of femoral bone.

The visualization of the results of the tests can have various forms. It may include full models, optionally created sections or structures isolated from the three-dimensional model. The use of the programs for analysis and three-dimensional reconstruction of the tomographical images, programs for solid geometry and biometrology in the conditions of implantation of endoprostheses constitute very useful equipment for optimal selection, because during the realization of the virtual procedures of the application of the endoprosheses, the geometrical system of the patient's anatomical structures is kept so there is the possibility to position the implant in the objective operational space. It is also possible to conduct a virtual separation of the anatomical structures, disconnection to the application and then return to the system which shows the real geometrical relations. In the geometrically modeled objects, in subsequent stages, the strength analysis with the loads and constraints put from the locomotion can be carried out.

\section{References}

[1] Bosker, B.H., Ettema, H.B., Verheyen, C.P., Castelein, R.M. (2009). Acetabular augmentation ring for recurrent dislocation stability rate after an average follow-up of 74 months. Int Orthop., 33(1), 49-52.

[2] Cech, O., Dzupa, V., Svatos, F. (2001). The uncemented cup of the CLS Spotorno joint endoprosthesis-5years' results. Acta Chir Orthop Traumatol., 68 (1), 10-17.

[3] DiGioia, A.M., Jaramaz, B., Blackwell, M. (1998). The Otto Aufranc Award. Image guided navigation system to measure intraoperatively acetabular implant alignment. Clin Orthop., 355, 8-22.

[4] Gandhi, R., Marchie, A. Farrokhyar, F., Nizar M. (2009). Computer navigation in total hip replacement a meta-analysis. Int Orthop., 33(3), 593-597.

[5] Hawi, N., Liodakis, E., O'Loughlin, P.F., Kabbani, A.R., Stüber, V., Petri, M., Stübig, T., Krettek, C., Citak, M. (2012). Progress towards intra-operative measurement of femoral antetorsion. Technol Health Care., 20(1), 57-63.

[6] Hisatome, T., Doi, H. (2011). Theoretically optimum position of the prosthesis in total hip arthroplasty to fulfill the severe range of motion criteria due to neck impingement. J Orthop Sci, 16(2), 229-237.

[7] Jaramaz, B., DiGioia, A.M., Blackwell, M., Nikau, C. (1998). Computer assisted measurement of cup placement in total hip replacement. Clin Orthop., 354, 70-81.

[8] Lewinnek, G.E., Lewis, J.L., Tarr, R. (1978). Compere CL and Zimmerman JR. Dislocations after total hip-replacement arthroplasties. J Bone Joint Surg Am., 60, 217-220.

[9] Li, Y.J., Yang, G.J., Zhang, L.C., Cai, C.Y., Wu, L.J. (2010). Influences of head/neck ratio and femoral antetorsion on the safe-zone of operative acetabular orientations in total hip arthroplasty. Chin $J$ Traumatol., 13(4), 206-211.

[10] Madej, T., Ryniewicz, A.M. (2010). The material-structure estimation of endoprostheses of hip joint in the numerical simulations. Engineering of Biomaterials, 95(14), 22-32.

[11] Ryniewicz, A. (2010). Accuracy assessment of shape mapping using computer tomography. Metrol. Meas. Syst., 17(3), 481-492.

[12] Ryniewicz, A.M. (2011). Identification, modeling and biotribology of human joints. Cracow, AGH University of Science and Technology Press.

[13] Ryniewicz, A.M., Madej, T. (2010). Preclinical tests of endoprostheses of hip joint using finite elements methods. Engineering of Biomaterials, 94(9), 14-23.

[14] Sendtner, E., Tibor, S., Winkler, R., Wörner, M., Grifka, J., Renkawitz, T. (2010). Stem torsion in total hip replacement. Acta Orthop., 81(5), 579-582.

[15] Sendtner, E., Müller, M., Winkler, R., Wörner, M., Grifka, J., Renkawitz, T. (2010). Femur first in hip arthroplasty-the concept of combined anteversion. Z Orthop Unfall. 148(2), 185-190. 